TAIWANESE JOURNAL OF MATHEMATICS

Vol. 15, No. 2, pp. 501-522, April 2011

This paper is available online at http://www.tjm.nsysu.edu.tw/

\title{
THE MULTIPLE HURWITZ ZETA FUNCTION AND THE MULTIPLE HURWITZ-EULER ETA FUNCTION
}

\author{
Junesang Choi and H. M. Srivastava*
}

\begin{abstract}
Almost eleven decades ago, Barnes introduced and made a systematic investigation on the multiple Gamma functions $\Gamma_{n}$. In about the middle of 1980s, these multiple Gamma functions were revived in the study of the determinants of Laplacians on the $n$-dimensional unit sphere $\mathbf{S}^{n}$ by using the multiple Hurwitz zeta functions $\zeta_{n}(s, a)$. In this paper, we first aim at presenting a generalized Hurwitz formula for $\zeta_{n}(s, a)$ together with its various special cases. Secondly, we give analytic continuations of multiple Hurwitz-Euler eta function $\eta_{n}(s, a)$ in two different ways. As a by-product of our second investigation, a relationship between $\eta_{n}(-\ell, a) \quad\left(\ell \in \mathbb{N}_{0}\right)$ and the generalized Euler polynomials $E_{\ell}^{(n)}(n-a)$ is also presented.
\end{abstract}

\section{Introduction and Preliminaries}

Barnes [4] (see also [1, 2, 3]) introduced and studied the generalized multiple Hurwitz zeta function $\zeta_{n}\left(s, a \mid w_{1}, \cdots, w_{n}\right)$ defined, for $\Re(s)>n$, by the following $n$-ple series:

$$
\zeta_{n}\left(s, a \mid w_{1}, \cdots, w_{n}\right):=\sum_{k_{1}, \cdots, k_{n}=0}^{\infty} \frac{1}{(a+\Omega)^{s}} \quad(\Re(s)>n ; n \in \mathbb{N}),
$$

where $\mathbb{N}$ denotes the set of positive integers,

$$
\Omega=k_{1} w_{1}+\cdots+k_{n} w_{n}
$$

Received September 7, 2009, accepted September 8, 2009.

Communicated by J. C. Yao.

2000 Mathematics Subject Classification: Primary 11M06, 11M99; Secondary 33B99.

Key words and phrases: Gamma function, Multiple Gamma functions, Riemann zeta function, Hurwitz (or generalized) zeta function, Multiple Hurwitz zeta function, Eta function, Multiple Hurwitz-Euler eta function, Taylor-Maclaurin series expansion, Generalized Bernoulli numbers and polynomials, Generalized Euler numbers and polynomials.

*Corresponding author. 
and the general conditions for $a$ and the parameters $w_{1}, \cdots, w_{n}$ are given in the work of Barnes [4] who used it in the study of the multiple Gamma functions $\Gamma_{n}$ (see [12, Section 1.3]). In about the middle of 1980s, the multiple Gamma functions $\Gamma_{n}$ were revived in the study of the determinants of Laplacians on the $n$-dimensional unit sphere $\mathbf{S}^{n}$ (see, e.g., $[5,8,10,11,13,14]$ ).

Here we consider only the following simple case of (1.1) when

$$
w_{j}=1 \quad(j=1, \cdots, n ; j, n \in \mathbb{N}) .
$$

Thus, for

$$
\mathbb{Z}_{0}^{-}:=\mathbb{Z} \backslash \mathbb{N} \quad(\mathbb{Z}:=\{0, \pm 1, \pm 2, \cdots\})
$$

and $\mathbb{C}$ the set of complex numbers, we write this special case of (1.1) as follows:

$$
\begin{aligned}
\zeta_{n}(s, a):= & \sum_{k_{1}, \cdots, k_{n}=0}^{\infty}\left(a+k_{1}+\cdots+k_{n}\right)^{-s} \\
& \left(\Re(s)>n ; n \in \mathbb{N} ; a \in \mathbb{C} \backslash \mathbb{Z}_{0}^{-}\right),
\end{aligned}
$$

which is also referred to as the n-ple (or, simply, the multiple) Hurwitz zeta function.

We first need to summarize some of the known (and useful) properties of the function $\zeta_{n}(s, a)$ in (1.2) (see, e.g., [12, Chapter 2]) with a view to making them readily and easily accessible for their application in our subsequent sections (especially in Section 2).

It is easy to see that

$$
\zeta_{1}(s, a)=\zeta(s, a)
$$

is the Hurwitz (or generalized) zeta function $\zeta(s, a)$ defined by

$$
\zeta(s, a):=\sum_{k=0}^{\infty}(k+a)^{-s} \quad\left(\Re(s)>1 ; a \in \mathbb{C} \backslash \mathbb{Z}_{0}^{-}\right),
$$

which, upon setting $a=1$, yields the Riemann zeta function $\zeta(s)$ defined by

$$
\zeta(s):= \begin{cases}\sum_{n=1}^{\infty} \frac{1}{n^{s}}=\frac{1}{1-2^{-s}} \sum_{n=1}^{\infty} \frac{1}{(2 n-1)^{s}} & (\Re(s)>1) \\ \frac{1}{\left(1-2^{1-s}\right)} \sum_{n=1}^{\infty} \frac{(-1)^{n-1}}{n^{s}} & (\Re(s)>0 ; s \neq 1) .\end{cases}
$$

Both the Riemann zeta function $\zeta(s)$ and the Hurwitz zeta function $\zeta(s, a)$ can be continued meromorphically to the whole complex $s$-plane, except for a simple pole only at $s=1$ with their respective residue 1, in many different ways (see also the recent investigations by Garg et al. [7] and Lin et al. [9]). 
An integral representation of $\zeta_{n}(s, a)$ is given by

$$
\Gamma(s) \zeta_{n}(s, a)=\int_{0}^{\infty} \frac{x^{s-1} e^{-a x}}{\left(1-e^{-x}\right)^{n}} d x \quad(\Re(s)=\sigma>n ; n \in \mathbb{N}) .
$$

As a way to extend $\zeta_{n}(s, a)$ to the half-plane on the left of the line $\sigma=\Re(s)=n$, we recall a contour integral as follows:

$$
I_{n}(s, a)=-\frac{1}{2 \pi i} \int_{C} \frac{(-z)^{s-1} e^{-a z}}{\left(1-e^{-z}\right)^{n}} d z \quad(a>0),
$$

which defines an entire function of $s$. Moreover, we have

$$
\zeta_{n}(s, a)=\Gamma(1-s) I_{n}(s, a) \quad(\Re(s)=\sigma>n ; n \in \mathbb{N}) .
$$

The contour $C$ is essentially a Hankel's loop (cf., e.g., Whittaker and Watson [15, p. 245]), which starts from $\infty$ along the upper side of the positive real axis, encircles the origin once in the positive (counter-clockwise) direction, and then returns to $\infty$ along the lower side of the positive real axis. In fact, the loop $C$ can be decomposed into three parts $C_{1}, C_{2}$ and $C_{3}$, where $C_{2}$ is a positively-oriented circle of radius $c<2 \pi$ centred at the origin, and $C_{1}$ and $C_{3}$ are the upper and lower edges of a cut in the complex $z$-plane along the positive real axis, traversed as given in Figure 1.

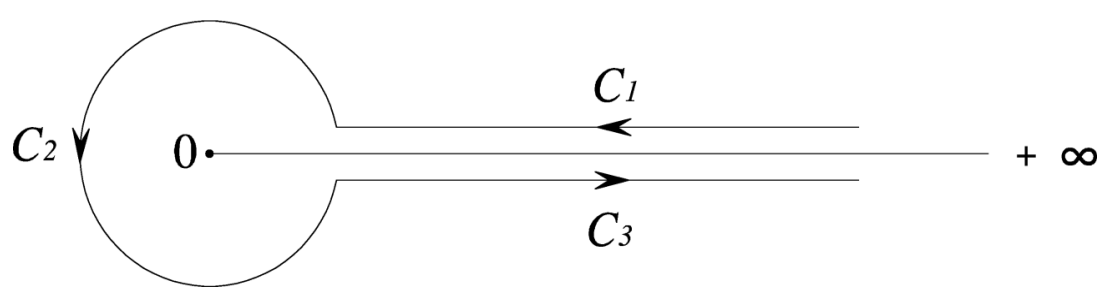

Fig. 1.

Since $I_{n}(s, a)$ is an entire function of $s$ for $a>0$, the only possible singularities of $\zeta_{n}(s, a)$ are the poles of $\Gamma(1-s)$. It is known that $\Gamma(1-s)$ has simple poles at $s \in \mathbb{N}$. But the series definition (1.2) shows that $\zeta_{n}(s, a)$ is analytic in

$$
\Re(s)=\sigma>n \quad(n \in \mathbb{N}),
$$

and so $s=1, \cdots, n$ are the only poles of $\zeta_{n}(s, a)$. If, on the other hand,

$$
\Re(s)=\sigma \leqq n \quad(n \in \mathbb{N}),
$$

we define $\zeta_{n}(s, a)$ by

$$
\zeta_{n}(s, a):=\Gamma(1-s) I_{n}(s, a)
$$


where $I_{n}(s, a)$ is given in (1.6). This equation (1.8) provides the analytic continuation of $\zeta_{n}(s, a)$ to the whole complex $s$-plane. The function $\zeta_{n}(s, a)$ defined by (1.8) is analytic for all $s$ except for simple poles at

$$
s=k \quad(1 \leqq k \leqq n ; n, k \in \mathbb{N}) .
$$

From (1.6) and (1.8) we can get the following relationship between $\zeta_{n}(s, a)$ and $B_{n}^{(\alpha)}(a)$ :

$$
\zeta_{n}(-\ell, a)=(-1)^{n} \frac{\ell !}{(n+\ell) !} B_{n+\ell}^{(n)}(a) \quad\left(\ell \in \mathbb{N}_{0}:=\mathbb{N} \cup\{0\}\right),
$$

where the generalized Bernoulli polynomials $B_{n}^{(\alpha)}(a)$ of degree $n$ in $a$ are defined by the generating function:

$$
\left(\frac{z}{e^{z}-1}\right)^{\alpha} e^{a z}=\sum_{n=0}^{\infty} B_{n}^{(\alpha)}(a) \frac{z^{n}}{n !} \quad\left(|z|<2 \pi ; 1^{\alpha}:=1\right)
$$

for arbitrary (real or complex) parameter $\alpha$. Clearly, we have

$$
B_{n}^{(\alpha)}(a)=(-1)^{n} B_{n}^{(\alpha)}(\alpha-a),
$$

so that

$$
B_{n}^{(\alpha)}(\alpha)=(-1)^{n} B_{n}^{(\alpha)}(0)=:(-1)^{n} B_{n}^{(\alpha)}
$$

in terms of the generalized Bernoulli numbers $B_{n}^{(\alpha)}$ defined by the generating function:

$$
\left(\frac{z}{e^{z}-1}\right)^{\alpha}=\sum_{n=0}^{\infty} B_{n}^{(\alpha)} \frac{z^{n}}{n !} \quad\left(|z|<2 \pi ; 1^{\alpha}:=1\right) .
$$

It is easily observed that

$$
B_{n}^{(1)}(a)=B_{n}(a) \quad \text { and } \quad B_{n}^{(1)}=B_{n} \quad\left(n \in \mathbb{N}_{0}\right),
$$

where $B_{n}(a)$ and $B_{n}$ are the familiar Bernoulli polynomials and Bernoulli numbers, respectively.

Setting $n=1$ in (1.9), we have the following well-known result:

$$
\zeta(-\ell, a)=-\frac{B_{\ell+1}(a)}{\ell+1} \quad\left(\ell \in \mathbb{N}_{0}\right) .
$$

where $\zeta(s, a)$ is the Hurwitz (or generalized) zeta function given in (1.3). 
Choi [6] (see also [12, Section 2.2]) presented another analytic continuation of $\zeta_{n}(s, a)$, which is different from the contour integral representation (1.8), by expressing it as a finite linear combination of the Hurwitz (or generalized) zeta functions $\zeta(s, a)$ as follows.

Proposition. The number of solutions of

$$
k_{1}+\cdots+k_{n}=k \quad\left(k \in \mathbb{N}_{0} ;\left(k_{1}, \cdots, k_{n}\right) \in \mathbb{N}_{0}{ }^{n}\right)
$$

is equal to the coefficient of $a^{k}$ in the following Taylor-Maclaurin series expansion of $(1-a)^{-n}$ :

$$
(1-a)^{-n}=\sum_{k=0}^{\infty}\left(\begin{array}{c}
-n \\
k
\end{array}\right)(-a)^{k}=\sum_{k=0}^{\infty}\left(\begin{array}{c}
k+n-1 \\
n-1
\end{array}\right) a^{k}
$$

Furthermore, the multiple Hurwitz zeta function in (1.2) can be expressed as the following simple series:

$$
\zeta_{n}(s, a)=\sum_{k=0}^{\infty}\left(\begin{array}{c}
k+n-1 \\
n-1
\end{array}\right)(a+k)^{-s} .
$$

For further analysis of (1.17), we recall the Stirling numbers of the first kind $s(n, \ell)$ defined by the following generating functions:

$$
a(a-1) \cdots(a-n+1)=\sum_{\ell=0}^{n} s(n, \ell) a^{\ell}
$$

and

$$
[\log (1+a)]^{\ell}=\ell ! \sum_{n=\ell}^{\infty} s(n, \ell) \frac{a^{n}}{n !} \quad(|a|<1) .
$$

It is found from (1.18) that the Pochhammer symbol $(a)_{n}$ defined by

$$
(a)_{n}:= \begin{cases}1 & (n=0) \\ a(a+1) \cdots(a+n-1) & (n \in \mathbb{N})\end{cases}
$$

can be easily expanded in terms of $s(n, \ell)$ :

$$
(a)_{n}=a(a+1) \cdots(a+n-1)=\sum_{\ell=0}^{n}(-1)^{n+\ell} s(n, \ell) a^{\ell},
$$


where the coefficient

$$
(-1)^{n+\ell} s(n, \ell)=|s(n, \ell)|:=|s|(n, \ell)
$$

is called the unsigned or absolute Stirling numbers of the first kind and represents the number of permutations of $n$ symbols which have exactly $\ell$ cycles. It is known that the Stirling numbers $s(n, \ell)$ satisfy the following recurrence relations:

$$
s(n+1, \ell)=s(n, \ell-1)-n s(n, \ell) \quad(n \geqq \ell \geqq 1)
$$

and

$$
\left(\begin{array}{l}
k \\
j
\end{array}\right) s(n, k)=\sum_{\ell=k-j}^{n-j}\left(\begin{array}{l}
n \\
\ell
\end{array}\right) s(n-\ell, j) s(\ell, k-j) \quad(n \geqq k \geqq j) .
$$

It is not difficult to see also that

$$
\begin{aligned}
& s(n, 0)=\left\{\begin{array}{ll}
1 & (n=0) \\
0 & (n \in \mathbb{N}),
\end{array} \quad s(n, n)=1,\right. \\
& s(n, 1)=(-1)^{n+1}(n-1) ! \quad \text { and } \quad s(n, n-1)=-\left(\begin{array}{l}
n \\
2
\end{array}\right) .
\end{aligned}
$$

Choi [6] observed that

$$
\left(\begin{array}{c}
k+n-1 \\
n-1
\end{array}\right)=\frac{1}{(n-1) !} \sum_{\ell=0}^{n-1}|s|(n, \ell+1) k^{\ell} .
$$

Substituting from (1.25) into (1.17) and using the following double-series identity:

$$
\sum_{\ell=0}^{n} \sum_{j=0}^{\ell} A_{\ell, j}=\sum_{j=0}^{n} \sum_{\ell=j}^{n} A_{\ell, j},
$$

Choi [6] found that $\zeta_{n}(s, a)$ is expressible as the following finite linear combination of the generalized zeta functions $\zeta(s, a)$ with polynomial coefficients in $a$ :

$$
\zeta_{n}(s, a)=\sum_{j=0}^{n-1} p_{n, j}(a) \zeta(s-j, a)
$$

where

$$
p_{n, j}(a)=\frac{1}{(n-1) !} \sum_{\ell=j}^{n-1}(-1)^{n+1-j}\left(\begin{array}{l}
\ell \\
j
\end{array}\right) s(n, \ell+1) a^{\ell-j} .
$$


We find that $p_{n, j}(a)$ in (1.28) is a polynomial in $a$ of degree $n-1-j$ with rational coefficients.

Since $\zeta(s, a)$ can be continued analytically to a meromorphic function having a simple pole at $s=1$ with its residue 1 , the representation (1.27) shows that $\zeta_{n}(s, a)$ is analytic for all $s$ except for simple poles only at $s=k(k=1, \cdots, n ; n \in \mathbb{N})$ with their respective residues given by

$$
\operatorname{Res}_{s=k} \zeta_{n}(s, a)=p_{n, k-1}(a) \quad(k=1, \cdots, n ; n \in \mathbb{N}) .
$$

The series for $\zeta_{n}(s, a)$ can be evaluated explicitly for the first few values of $n$ :

$$
\begin{aligned}
\zeta_{2}(s, a)= & (1-a) \zeta(s, a)+\zeta(s-1, a), \\
\zeta_{3}(s, a)= & \frac{1}{2}\left(a^{2}-3 a+2\right) \zeta(s, a)+\left(\frac{3}{2}-a\right) \zeta(s-1, a)+\frac{1}{2} \zeta(s-2, a), \\
\zeta_{4}(s, a)= & \frac{1}{6}\left\{\left(-a^{3}+6 a^{2}-11 a+6\right) \zeta(s, a)+\left(3 a^{2}-12 a+11\right) \zeta(s-1, a)\right. \\
& -(3 a-6) \zeta(s-2, a)+\zeta(s-3, a)\} .
\end{aligned}
$$

On the other hand, it is found from (1.21) that

$$
\left(\begin{array}{c}
k+n-1 \\
n-1
\end{array}\right)=\frac{(k+1)_{n-1}}{(n-1) !}=\frac{1}{(n-1) !} \sum_{\ell=0}^{n-1}|s|(n-1, \ell)(k+1)^{\ell} .
$$

Setting (1.31) in (1.17) and using the same technique as in [6] yields a seemingly different expression from (1.27):

$$
\zeta_{n}(s, a)=\sum_{j=0}^{n-1} q_{n, j}(a) \zeta(s-j, a),
$$

where

$$
q_{n, j}(a)=\frac{1}{(n-1) !} \sum_{\ell=j}^{n-1}(-1)^{n+\ell-1}\left(\begin{array}{l}
\ell \\
j
\end{array}\right) s(n-1, \ell)(1-a)^{\ell-j} .
$$

We show that (1.28) and (1.33) are equivalent. Since $s(n, 0)=0 \quad(n \in \mathbb{N})$, it follows from (1.21) that

$$
a(a+1) \cdots(a+n-1)=\sum_{\ell=1}^{n}(-1)^{n+\ell} s(n, \ell) a^{\ell},
$$


which, upon dividing by $a$, yields

$$
(a+1)_{n-1}=(a+1) \cdots(a+n-1)=\sum_{\ell=1}^{n}(-1)^{n+\ell} s(n, \ell) a^{\ell-1} .
$$

Now, using (1.21) for the left-hand side and letting $\ell-1=\ell^{\prime}$ for the right-hand side, and then dropping the prime on $\ell^{\prime}$, we are led to the following identity:

$$
\begin{aligned}
\sum_{\ell=0}^{n-1} & (-1)^{n+1+\ell} s(n-1, \ell)(a+1)^{\ell} \\
& =\sum_{\ell=0}^{n-1}(-1)^{n+1+\ell} s(n, \ell+1) a^{\ell} \quad(n \in \mathbb{N}),
\end{aligned}
$$

which does indeed show that (1.28) and (1.33) are equivalent.

In this paper, we first extend the Hurwitz formula (2.9) for $\zeta(s, a)$ by presenting a generalized Hurwitz formula for $\zeta_{n}(s, a)$ and consider its various special cases. Secondly, we investigate and give the analytic continuation of the multiple HurwitzEuler eta function $\eta_{n}(s, a)$ in two different ways. As a by-product of the second investigation, a relationship between $\eta_{n}(-\ell, a)\left(\ell \in \mathbb{N}_{0}\right)$ and the generalized Euler polynomials $E_{\ell}^{(n)}(n-a)$ of Definition 3 below is also provided.

\section{A Generalized Hurwitz Formula for $\zeta_{n}(s, a)$}

The series expression (1.2) for $\zeta_{n}(s, a)$ has a meaning whenever

$$
\Re(s)=\sigma>n \quad(s=\sigma+i t ; n \in \mathbb{N}) .
$$

We present here another series representation for $\zeta_{n}(s, a)$, which is valid in the half-plane $\Re(s)=\sigma<0$.

Theorem 1. The following explicit representation holds true:

$$
\begin{gathered}
\zeta_{n}(1-s, a)=\Gamma(s) \sum_{k=1}^{\infty}\{\mathcal{R}(n ; k)+\mathcal{R}(n ;-k)\} \\
(0<a \leqq n ; n \in \mathbb{N} ; \sigma=\Re(s)>1),
\end{gathered}
$$

where $\mathcal{R}(n ; k)$ denotes the residue of the function

$$
h(n, a, s ; z):=\frac{(-z)^{-s} e^{(n-a) z}}{\left(e^{z}-1\right)^{n}}
$$

at $z=2 k \pi i \quad(k \in \mathbb{Z} \backslash\{0\})$. 
We first prove the following Lemma which will be used in the proof of the assertion (2.1) of Theorem 1.

Lemma. Let $P(\delta)$ denote the region that remains when we remove from the complex z-plane all open circular disks of radius $\delta \quad(0<\delta<\pi)$ with centers at $z=2 k \pi i \quad(k \in \mathbb{Z})$. Then the function

$$
f(z)=\frac{e^{-a z}}{\left(1-e^{-z}\right)^{n}}=\frac{e^{(n-a) z}}{\left(e^{z}-1\right)^{n}} \quad(0<a \leqq n ; n \in \mathbb{N})
$$

is bounded in $P(\delta)$ with the bound depending upon $\delta$ and $n)$.

Proof. Write $z=x+i y$ and consider the punctured rectangle $Q(\delta)$ given by

$$
Q(\delta)=\{z: z \in \mathbb{C},|x| \leqq 1,|y| \leqq \pi \quad \text { and } \quad|z| \geqq \delta\} .
$$

Since $f(z)$ is a continuous function on the compact punctured rectangle $Q(\delta)$, $f$ is bounded on $Q(\delta)$. Moreover, since $|f(z+2 \pi i)|=|f(z)|$, i.e., since $|f(z)|$ is a periodic function with a purely imaginary period $2 \pi i, f$ is bounded in the punctured infinite strip $S(\delta)$ given by

$$
S(\delta)=\{z: z \in \mathbb{C},|x| \leqq 1 \quad \text { and }|z-2 k \pi i| \geqq \delta \quad(k \in \mathbb{Z})\} .
$$

We next show that $f$ is bounded outside the strip $S(\delta)$. Suppose that $|x| \geqq 1$ and consider

$$
|f(z)|=\left|\frac{e^{(n-a) z}}{\left(e^{z}-1\right)^{n}}\right|=\frac{e^{(n-a) x}}{\left|\left(e^{z}-1\right)^{n}\right|} \leqq \frac{e^{(n-a) x}}{\left|e^{x}-1\right|^{n}} .
$$

When $x \geqq 1$, we have

$$
\left|e^{x}-1\right|=e^{x}-1 \quad \text { and } \quad e^{(n-a) x} \leqq e^{n x},
$$

so that

$$
|f(z)| \leqq \frac{e^{n x}}{\left(e^{x}-1\right)^{n}}=\frac{1}{\left(1-e^{-x}\right)^{n}} \leqq \frac{1}{\left(1-e^{-1}\right)^{n}}=\frac{e^{n}}{(e-1)^{n}} .
$$

On the other hand, when $x \leqq-1$, we have

$$
\left|e^{x}-1\right|=1-e^{x} \quad \text { and } \quad e^{(n-a) x} \leqq 1,
$$

so that

$$
|f(z)| \leqq \frac{1}{\left(1-e^{x}\right)^{n}} \leqq \frac{1}{\left(1-e^{-1}\right)^{n}}=\frac{e^{n}}{(e-1)^{n}}
$$


Hence

$$
|f(z)| \leqq \frac{e^{n}}{(e-1)^{n}} \quad(|\Re(z)| \geqq 1),
$$

which completes the proof of the Lemma.

Proof of Theorem 1. Consider the function

$$
F_{N}(s, a)=-\frac{1}{2 \pi i} \int_{C(N)} \frac{(-z)^{s-1} e^{-a z}}{\left(1-e^{-z}\right)^{n}} d z \quad(N \in \mathbb{N})
$$

where

$$
C(N):=C_{1} \cup C_{2} \cup C_{3} \cup C_{R} \quad(R:=(2 N+1) \pi ; \quad N \in \mathbb{N})
$$

is a simple closed contour as shown in Figure 2. In particular,

$$
C_{R}: z=(2 N+1) \pi e^{i \theta} \quad(\theta \text { varies from } 2 \pi \text { to } 0 ; N \in \mathbb{N}) .
$$

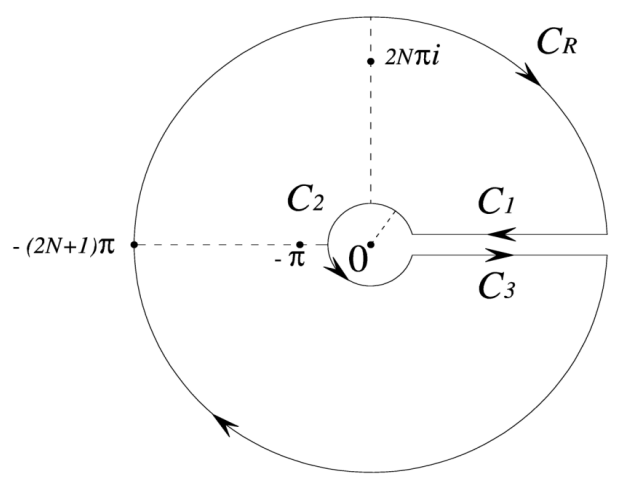

Fig. 2.

We first prove that

$$
\lim _{N \rightarrow \infty} F_{N}(s, a)=I_{n}(s, a) \quad(\Re(s)=\sigma<0),
$$

where $I_{n}(s, a)$ is given by (1.6). To do this, in view of (1.6), it suffices to show that the integral along the outer circle $C_{R}$ tends to 0 as $N \rightarrow \infty$. On $C_{R}$, we have

$$
\left|(-z)^{s-1}\right|=\left|(-R)^{s-1} e^{i \theta(s-1)}\right|=R^{\sigma-1} e^{-t \theta} \leqq R^{\sigma-1} e^{2 \pi|t|} \quad(s=\sigma+i t) .
$$

Let $M$ be the bound for the function

$$
\left|\frac{e^{(n-a) z}}{\left(e^{z}-1\right)^{n}}\right|,
$$


which is implied by the above Lemma. Then it is seen that

$$
\left|\int_{C_{R}} \frac{(-z)^{s-1} e^{-a z}}{\left(1-e^{-z}\right)^{n}} d z\right| \leqq 2 \pi M e^{2 \pi|t|} R^{\sigma} \rightarrow 0 \quad(R \rightarrow \infty ; \sigma=\Re(s)<0) .
$$

Hence, upon replacing $s$ by $1-s$ in (2.3), we find that

$$
\lim _{N \rightarrow \infty} F_{N}(1-s, a)=I_{n}(1-s, a) \quad(\Re(s)=\sigma>1) .
$$

The integrand in $F_{N}(1-s, a)$ has poles of order $n$ at $z=2 k \pi i \quad(k \in \mathbb{Z} \backslash\{0\})$ and so we have

$$
\begin{aligned}
\mathcal{R}(n ; k) & :=\operatorname{Res}_{z=2 k \pi i} h(n, a, s ; z) \\
& =\frac{1}{(n-1) !} \lim _{z \rightarrow 2 k \pi i} \frac{d^{n-1}}{d z^{n-1}}\left\{(z-2 k \pi i)^{n} h(n, a, s ; z)\right\},
\end{aligned}
$$

where, in particular,

$$
\begin{gathered}
\mathcal{R}(1 ; k)=\frac{e^{-2 k \pi i a}}{(-2 k \pi i)^{s}}, \\
\mathcal{R}(2 ; k)=\frac{s e^{-2 k \pi i a}}{(-2 k \pi i)^{s+1}}+\frac{(1-a) e^{-2 k \pi i a}}{(-2 k \pi i)^{s}},
\end{gathered}
$$

and

$$
\begin{gathered}
\mathcal{R}(3 ; k)=\frac{1}{2} s(s+1) \frac{e^{-2 k \pi i a}}{(-2 k \pi i)^{s+2}}+\left(\frac{3}{2}-a\right) \frac{s e^{-2 k \pi i a}}{(-2 k \pi i)^{s+1}} \\
+\frac{1}{2}\left(a^{2}-3 a+2\right) \frac{e^{-2 k \pi i a}}{(-2 k \pi i)^{s}} .
\end{gathered}
$$

The proof of Theorem 1 is thus completed.

By applying (2.6), (2.7) and (2.8) to (2.1), we obtain Corollary 1 below.

Corollary 1. The following relationships hold true:

$$
\begin{aligned}
& \zeta(1-s, a)=\frac{\Gamma(s)}{(2 \pi)^{s}}\left\{e^{-\frac{1}{2} \pi i s} L(a, s)+e^{\frac{1}{2} \pi i s} L(-a, s)\right\} \\
& (0<a \leqq 1 \text { and } \sigma=\Re(s)>1 ; 0<a<1 \text { and } \sigma=\Re(s)>0),
\end{aligned}
$$




$$
\begin{aligned}
\zeta_{2}(1- & s, a) \\
= & \frac{\Gamma(s+1)}{(2 \pi)^{s+1}}\left\{e^{\frac{1}{2} \pi i(s+1)} L(-a, s+1)+e^{-\frac{1}{2} \pi i(s+1)} L(a, s+1)\right\} \\
& +(1-a) \frac{\Gamma(s)}{(2 \pi)^{s}}\left\{e^{\frac{1}{2} \pi i s} L(-a, s)+e^{-\frac{1}{2} \pi i s} L(a, s)\right\} \\
& (0<a \leqq 1 \text { and } \sigma=\Re(s)>1 ; 0<a<1 \text { and } \sigma=\Re(s)>0)
\end{aligned}
$$

and

$$
\begin{aligned}
\zeta_{3}(1 & -s, a) \\
= & \frac{\Gamma(s+2)}{2(2 \pi)^{s+2}}\left\{e^{\frac{1}{2} \pi i(s+2)} L(-a, s+2)+e^{-\frac{1}{2} \pi i(s+2)} L(a, s+2)\right\} \\
& +\left(\frac{3}{2}-a\right) \frac{\Gamma(s+1)}{(2 \pi)^{s+1}}\left\{e^{\frac{1}{2} \pi i(s+1)} L(-a, s+1)+e^{-\frac{1}{2} \pi i(s+1)} L(a, s+1)\right\} \\
& +\frac{1}{2}\left(a^{2}-3 a+2\right) \frac{\Gamma(s)}{(2 \pi)^{s}}\left\{e^{\frac{1}{2} \pi i s} L(-a, s)+e^{-\frac{1}{2} \pi i s} L(a, s)\right\} \\
& (0<a \leqq 1 \text { and } \sigma=\Re(s)>1 ; 0<a<1 \text { and } \sigma=\Re(s)>0),
\end{aligned}
$$

where $L(a, s)$ denotes the Dirichlet series defined by

$$
L(a, s):=\sum_{n=1}^{\infty} \frac{e^{2 n \pi i a}}{n^{s}} \quad(a \in \mathbb{R} ; \sigma=\Re(s)>1) .
$$

The relationship (2.9) is, in fact, Hurwitz's formula for $\zeta(s, a)$ (see, e.g., [12, p. 89, Eq. 2.2(6)]). Furthermore, the Dirichlet series $L(a, s)$ defined by (2.12) is often referred to as the periodic (or Lerch) zeta function. Indeed the Dirichlet series in (2.12) is a periodic function of $a$ with period 1 and

$$
L(1, s)=\zeta(s),
$$

in terms of the Riemann zeta function $\zeta(s)$. The series in (2.12) converges absolutely for

$$
\sigma=\Re(s)>1 .
$$

Moreover, if $a \notin \mathbb{Z}$, the series in (2.12) can also be seen to converge conditionally for

$$
\sigma=\Re(s)>0 .
$$

Consequently, the formulas (2.9) to (2.11) are all valid for

$$
\sigma=\Re(s)>0 \quad(a \neq 1) .
$$


We observe that the function $L(a, s)$ in (2.12) is a linear combination of the Hurwitz zeta functions when $a$ is a rational number. Since the set $\mathbb{N}$ of positive integers is a disjoint union of the residue classes $\bmod q(q \in \mathbb{N})$ as follows:

$$
\mathbb{N}=\bigcup_{r=1}^{q}\left\{k q+r \mid k \in \mathbb{N}_{0}\right\}
$$

we are led easily to the familiar series identity:

$$
\sum_{n=1}^{\infty} \Theta(n)=\sum_{r=1}^{q} \sum_{k=0}^{\infty} \Theta(k q+r) \quad(q \in \mathbb{N}),
$$

if the involved series is absolutely convergent.

Setting

$$
a=\frac{p}{q} \quad(1 \leqq p \leqq q ; p, q \in \mathbb{N})
$$

in (2.12) and using the series identity (2.13), we see, for $\Re(s)=\sigma>1$, that

$$
L\left(\frac{p}{q}, s\right)=\frac{1}{q^{s}} \sum_{r=1}^{q} \exp \left(\frac{2 \pi i r p}{q}\right) \zeta\left(s, \frac{r}{q}\right) .
$$

Therefore, if we take $a=\frac{p}{q}$ in the formulas (2.9) to (2.11), we obtain Corollary 2 below.

Corollary 2. Each of the following relationships holds true:

$$
\begin{gathered}
\zeta\left(1-s, \frac{p}{q}\right)=\frac{2 \Gamma(s)}{(2 \pi q)^{s}} \sum_{r=1}^{q} \cos \left(\frac{\pi s}{2}-\frac{2 \pi r p}{q}\right) \zeta\left(s, \frac{r}{q}\right) \\
\quad(1 \leqq p \leqq q ; p, q \in \mathbb{N} ; \sigma=\Re(s)>1), \\
\zeta_{2}\left(1-s, \frac{p}{q}\right) \\
=\left(1-\frac{p}{q}\right) \frac{2 \Gamma(s)}{(2 \pi q)^{s}} \sum_{r=1}^{q} \cos \left(\frac{\pi s}{2}-\frac{2 \pi r p}{q}\right) \zeta\left(s, \frac{r}{q}\right) \\
+\frac{2 \Gamma(s+1)}{(2 \pi q)^{s+1}} \sum_{r=1}^{q} \cos \left(\frac{\pi(s+1)}{2}-\frac{2 \pi r p}{q}\right) \zeta\left(s+1, \frac{r}{q}\right) \\
(1 \leqq p \leqq q ; p, q \in \mathbb{N} ; \sigma=\Re(s)>1)
\end{gathered}
$$

and 


$$
\begin{aligned}
\zeta_{3}(1- & \left.s, \frac{p}{q}\right) \\
= & {\left[\left(\frac{p}{q}\right)^{2}-\frac{3 p}{q}+2\right] \frac{\Gamma(s)}{(2 \pi q)^{s}} \sum_{r=1}^{q} \cos \left(\frac{\pi s}{2}-\frac{2 \pi r p}{q}\right) \zeta\left(s, \frac{r}{q}\right) } \\
& +\left(3-\frac{2 p}{q}\right) \frac{\Gamma(s+1)}{(2 \pi q)^{s+1}} \sum_{r=1}^{q} \cos \left(\frac{\pi(s+1)}{2}-\frac{2 \pi r p}{q}\right) \zeta\left(s+1, \frac{r}{q}\right) \\
& +\frac{\Gamma(s+2)}{(2 \pi q)^{s+2}} \sum_{r=1}^{q} \cos \left(\frac{\pi(s+2)}{2}-\frac{2 \pi r p}{q}\right) \zeta\left(s+2, \frac{r}{q}\right) \\
& (1 \leqq p \leqq q ; p, q \in \mathbb{N} ; \sigma=\Re(s)>1) .
\end{aligned}
$$

The relationship (2.14) is the familiar Rademacher's formula (see, e.g., [12, p. 90, Eq. 2.2(8)]). Moreover, each of the formulas (2.14) to (2.16) holds true, by the principle of analytic continuation, for all admissible values of $s \in \mathbb{C}$.

\section{Multiple Hurwitz-Euler Eta Function}

The alternating Hurwitz zeta function (or, equivalently, Hurwitz-Euler eta function) $\eta(s, a)$ is defined by

$$
\eta(s, a)=\sum_{k=0}^{\infty} \frac{(-1)^{k}}{(k+a)^{s}} \quad(\Re(s)>0 ; a>0) .
$$

The special case of (3.1) when $a=1$, denoted by

$$
\eta(s, 1):=\eta(s)=\sum_{k=1}^{\infty} \frac{(-1)^{k-1}}{k^{s}} \quad(\Re(s)>0),
$$

is called the Dirichlet eta function (or the alternating Riemann zeta function).

It is known that $\eta(s, a)$ (and so also $\eta(s)$ ) can be continued analytically to the whole complex $s$-plane. Clearly, therefore, each of the functions $\eta(s, a)$ and $\eta(s)$ is an entire function of $s \in \mathbb{C}$.

The multiple Hurwitz-Euler eta function $\eta_{n}(s, a)$ is defined by

(3.3) $\eta_{n}(s, a):=\sum_{k_{1}, \cdots, k_{n}=0}^{\infty} \frac{(-1)^{k_{1}+\cdots+k_{n}}}{\left(a+k_{1}+\cdots+k_{n}\right)^{s}} \quad(\Re(s)>0 ; a>0 ; n \in \mathbb{N})$.

By using the same process as in getting (1.32), we find that $\eta_{n}(s, a)$ is expressible as a finite linear combination of the alternating Hurwitz zeta function $\eta(s, a)$ with polynomials in $a$ as the coefficients. 
Theorem 2. The following relationship holds true:

$$
\eta_{n}(s, a)=\sum_{j=0}^{n-1} q_{n, j}(a) \eta(s-j, a),
$$

where the polynomials $q_{n, j}(a)$ are given in (1.33).

It follows from Theorem 2 that, since $\eta(s, a)$ can be continued analytically as an entire function of $s \in \mathbb{C}, \eta_{n}(s, a)$ can be continued analytically as an entire function on $\mathbb{C}$.

By using the familiar relation:

$$
\frac{\Gamma(s)}{p^{s}}=\int_{0}^{\infty} e^{-p t} t^{s-1} d t \quad(\Re(s)>0 ; \Re(p)>0),
$$

we obtain an integral representation of $\eta_{n}(s, a)$ given by Theorem 3 below.

Theorem 3. The following relationship holds true:

$$
\Gamma(s) \eta_{n}(s, a)=\int_{0}^{\infty} \frac{x^{s-1} e^{-a x}}{\left(1+e^{-x}\right)^{n}} d x \quad(\Re(s)>0 ; n \in \mathbb{N}) .
$$

By slightly modifying the method described in [12, p. 81], we can extend the integral representation (3.6) to all complex $s=\sigma+i t$ with $\sigma>0$. Indeed, we note that both functions on the left-hand side of (3.6) are analytic for $\sigma>0$. To show that the right-hand dise of (3.6) is also analytic, we first assume that

$$
\delta \leqq \sigma \leqq c \quad(c>0 ; \delta>0)
$$

and write

$$
\begin{aligned}
\int_{0}^{\infty}\left|\frac{e^{-a t} t^{s-1}}{\left(1+e^{-t}\right)^{n}}\right| d t & =\int_{0}^{\infty} \frac{e^{-a t} t^{\sigma-1}}{\left(1+e^{-t}\right)^{n}} d t \\
& =\left(\int_{0}^{1}+\int_{1}^{\infty}\right) \frac{e^{-a t} t^{\sigma-1}}{\left(1+e^{-t}\right)^{n}} d t
\end{aligned}
$$

If $0 \leqq t \leqq 1$, we have

$$
t^{\sigma-1} \leqq t^{\delta-1}
$$

And, if $t \geqq 1$, we have

$$
t^{\sigma-1} \leqq t^{c-1}
$$

Moreover, since

$$
e^{t} \geqq 1 \quad(t \geqq 0),
$$


we have

$$
\begin{aligned}
\int_{0}^{1} \frac{e^{-a t} t^{\sigma-1}}{\left(1+e^{-t}\right)^{n}} d t & \leqq \int_{0}^{1} \frac{e^{(n-a) t} t^{\delta-1}}{\left(e^{t}+1\right)^{n}} d t \leqq \frac{1}{2^{n}} \int_{0}^{1} e^{(n-a) t} t^{\delta-1} d t \\
& \leqq \begin{cases}e^{n-a} \int_{0}^{1} t^{\delta-1} d t=\frac{e^{n-a}}{\delta} & (0<a \leqq n) \\
\int_{0}^{1} t^{\delta-1} d t=\frac{1}{\delta} & (a>n)\end{cases}
\end{aligned}
$$

and

$$
\int_{1}^{\infty} \frac{e^{-a t} t^{\sigma-1}}{\left(1+e^{-t}\right)^{n}} d t \leqq \int_{0}^{\infty} \frac{e^{-a t} t^{c-1}}{\left(1+e^{-t}\right)^{n}} d t=\Gamma(c) \eta_{n}(c, a) .
$$

This shows that the integral in (3.6) converges uniformly in every strip given by

$$
\delta \leqq \sigma \leqq c \quad(\delta>0)
$$

and, therefore, it represents an analytic function in every such strip; hence also in the half-plane $\sigma>0$. Consequently, by the principle of analytic continuation, (3.6) holds true for all $s$ with $\Re(s)=\sigma>0$.

In order to extend $\eta_{n}(s, a)$ to the half-plane on the left of the line $\sigma=0$, we derive another representation in terms of a contour integral. The contour $C$ is essentially the same as in (1.6) or in Figure 1. Here $C_{1}$ and $C_{2}$ are the same as in Figure 1. But the radius $c$ of the positively-oriented circle $C_{2}$ should be constrained by $c<\pi$. Thus we can use the following parameterizations:

$$
-z=r e^{-\pi i} \text { on } C_{1}
$$

and

$$
-z=r e^{\pi i} \quad \text { on } \quad C_{3}
$$

where $r$ varies from $c$ to $\infty$.

Theorem 4. If $a>0$, then the function defined by the following contour integral:

$$
J_{n}(s, a)=-\frac{1}{2 \pi i} \int_{C} \frac{(-z)^{s-1} e^{-a z}}{\left(1+e^{-z}\right)^{n}} d z
$$

is an entire function of s. Furthermore

$$
\eta_{n}(s, a)=\Gamma(1-s) J_{n}(s, a) \quad(\Re(s)=\sigma>0) .
$$

Proof. Here $(-z)^{s}$ means $r^{s} e^{-\pi i s}$ on $C_{1}$ and $r^{s} e^{\pi i s}$ on $C_{3}$. We consider an arbitrary compact disk $|s| \leqq M$ and prove that the integrals along $C_{1}$ and $C_{3}$ 
converge uniformly on every such disk. Since the integrand in (3.7) is an entire function of $s$, this will prove that $J_{n}(s, a)$ is also an entire function of $s$ for $a>0$. Along $C_{1}$ we have, for $r \geqq 1$,

$$
\left|(-z)^{s-1}\right|=r^{\sigma-1}\left|e^{-\pi i(\sigma-1+i t)}\right|=r^{\sigma-1} e^{\pi t} \leqq r^{M-1} e^{\pi M},
$$

since $|s| \leqq M$. Similarly, along $C_{3}$ we have, for $r \geqq 1$,

$$
\left|(-z)^{s-1}\right|=r^{\sigma-1}\left|e^{\pi i(\sigma-1+i t)}\right|=r^{\sigma-1} e^{-\pi t} \leqq r^{M-1} e^{\pi M} .
$$

Hence, on either $C_{1}$ or $C_{3}$, we have, for $r \geqq 1$,

$$
\left|\frac{(-z)^{s-1} e^{-a z}}{\left(1+e^{-z}\right)^{n}}\right| \leqq \frac{r^{M-1} e^{\pi M} e^{-a r}}{\left(1-e^{-r}\right)^{n}} \leqq \frac{e^{\pi M}}{\left(1-e^{-1}\right)^{n}} \cdot r^{M-1} \cdot e^{-a r} .
$$

But the following integral:

$$
\int_{c}^{\infty} r^{M-1} e^{-a r} d r
$$

converges if $c>0$; this shows that the integrals along $C_{1}$ and $C_{3}$ converge uniformly on every compact disk $|s| \leqq M$, and hence $J_{n}(s, a)$ is an entire function of $s$. To prove (3.8), we write

$$
-2 \pi i J_{n}(s, a)=\left(\int_{C_{1}}+\int_{C_{2}}+\int_{C_{3}}\right)(-z)^{s-1} g(-z) d z,
$$

where

$$
g(-z)=\frac{e^{-a z}}{\left(1+e^{-z}\right)^{n}} .
$$

On $C_{1}$ and $C_{3}$ we have $g(-z)=g(-r)$, and on $C_{2}$ we write $-z=c e^{i \theta}$, where $\theta$ varies from $2 \pi$ to 0 . This yields

$$
\begin{aligned}
-2 \pi i J_{n}(s, a)= & \int_{\infty}^{c} r^{s-1} e^{-\pi i(s-1)} g(-r) d r \\
& -i \int_{2 \pi}^{0} c^{s-1} e^{(s-1) i \theta} c e^{i \theta} g\left(c e^{i \theta}\right) d \theta \\
& +\int_{c}^{\infty} r^{s-1} e^{\pi i(s-1)} g(-r) d r \\
= & -2 i \sin (\pi s) \int_{c}^{\infty} r^{s-1} g(-r) d r-i c^{s} \int_{2 \pi}^{0} e^{i s \theta} g\left(c e^{i \theta}\right) d \theta .
\end{aligned}
$$

Dividing both sides of (3.9) by $-2 i$, we obtain

$$
\pi J_{n}(s, a)=\sin (\pi s) I_{1}(s, c)+I_{2}(s, c),
$$


where

$$
I_{1}(s, c)=\int_{c}^{\infty} r^{s-1} g(-r) d r \quad \text { and } \quad I_{2}(s, c)=\frac{c^{s}}{2} \int_{2 \pi}^{0} e^{i s \theta} g\left(c e^{i \theta}\right) d \theta .
$$

We now let $c \rightarrow 0$. In view of (3.6), we thus find that

$$
\lim _{c \rightarrow 0} I_{1}(s, c)=\int_{0}^{\infty} \frac{r^{s-1} e^{-a r}}{\left(1-e^{-r}\right)^{n}} d r=\Gamma(s) \eta_{n}(s, a) \quad(\sigma=\Re(s)>0) .
$$

We next show that

$$
\lim _{c \rightarrow 0} I_{2}(s, c)=0 .
$$

To do this, we note that $g(-z)$ is analytic in $|z|<\pi$. Hence $g(-z)$ is bounded there, that is,

$$
|g(-z)| \leqq A \quad(|z|=c<\pi ; \quad A>0)
$$

for some positive constant $A$. This leads us to the following two-sided inequalities:

$$
\left|I_{2}(s, c)\right| \leqq \frac{c^{\sigma}}{2} \int_{0}^{2 \pi} e^{-t \theta} \cdot A d \theta \leqq \pi A e^{2 \pi|t|} c^{\sigma} \quad(A>0) .
$$

If $\sigma>0$ and $c \rightarrow 0$, we find that $I_{2}(s, c) \rightarrow 0$. Hence

$$
\pi J_{n}(s, a)=\sin (\pi s) \Gamma(s) \eta_{n}(s, a),
$$

which, by means of the following well-known relation:

$$
\Gamma(s) \Gamma(1-s)=\frac{\pi}{\sin (\pi s)} \quad(s \in \mathbb{C} \backslash \mathbb{Z}),
$$

is seen to be equivalent to (3.8).

In the equation (3.8), valid for $\sigma>0$, the function $J_{n}(s, a)$ is an entire function of $s$, and $\Gamma(1-s)$ is analytic for every complex $s(s \in \mathbb{C} \backslash \mathbb{N})$. We, therefore, can use this equation to define $\eta_{n}(s, a)$ for

$$
\sigma=\Re(s) \leqq 0,
$$

that is, outside

$$
\sigma=\Re(s)>0
$$

as desired.

Definition 1. For

$$
\Re(s)=\sigma \leqq 0,
$$


we define the function $\eta_{n}(s, a)$ by

$$
\eta_{n}(s, a):=\Gamma(1-s) J_{n}(s, a),
$$

where $J_{n}(s, a)$ is given in (3.7).

Clearly, the equation (3.12) provides an analytic continuation of $\eta_{n}(s, a)$ to the whole complex $s$-plane.

Just as in (1.9), in order to get a relationship between $\eta_{n}(s, a)$ and the generalized Euler polynomials $E_{n}^{(\alpha)}(a)$, we recall the following definitions.

Definition 2. The classical Euler polynomials $E_{k}(a)$ and the classical Euler numbers $E_{k}$ are defined by the following generating functions (see, e.g., [12, pp. 63-66]):

$$
\frac{2 e^{a z}}{e^{z}+1}=\sum_{k=0}^{\infty} E_{k}(a) \frac{z^{k}}{k !} \quad(|z|<\pi)
$$

and

$$
\frac{2 e^{z}}{e^{2 z}+1}=\operatorname{sech} z=\sum_{k=0}^{\infty} E_{k} \frac{z^{k}}{k !} \quad\left(|z|<\frac{\pi}{2}\right)
$$

respectively.

Definition 3. For an arbitrary (real or complex) parameter $\alpha$, the generalized Euler polynomials $E_{k}^{(\alpha)}(a)$ and the generalized Euler numbers $E_{k}^{(\alpha)}$ are defined by the generating functions:

$$
\left(\frac{2}{e^{z}+1}\right)^{\alpha} e^{a z}=\sum_{k=0}^{\infty} E_{k}^{(\alpha)}(a) \frac{z^{k}}{k !} \quad\left(|z|<\pi ; 1^{\alpha}:=1\right)
$$

and

$$
\left(\frac{2 e^{z}}{e^{2 z}+1}\right)^{\alpha}=\sum_{k=0}^{\infty} E_{k}^{(\alpha)} \frac{z^{k}}{k !} \quad\left(|z|<\frac{\pi}{2} ; 1^{\alpha}:=1\right),
$$

respectively. Clearly, we find from Definitions 2 and 3 that

$$
E_{k}^{(1)}(a)=E_{k}(a) \quad \text { and } \quad E_{k}^{(1)}=E_{k} \quad\left(k \in \mathbb{N}_{0}\right) .
$$

Setting

$$
s=-\ell \quad\left(\ell \in \mathbb{N}_{0}\right)
$$


in (3.12), we obtain

$$
\eta_{n}(-\ell, a)=\ell ! J_{n}(-\ell, a)
$$

where

$$
J_{n}(-\ell, a)=-\frac{1}{2 \pi i} \int_{C} \frac{(-z)^{-\ell-1} e^{(n-a) z}}{\left(e^{z}+1\right)^{n}} d z \quad\left(\ell \in \mathbb{N}_{0}\right) .
$$

The integrand in the contour integral (3.18) for $J_{n}(-\ell, a)$ takes on the same values on both $C_{1}$ and $C_{3}$, but with opposite signs. Hence the integrals along $C_{1}$ and $C_{3}$ cancel. Thus, In view of (3.15), we find that

$$
\begin{aligned}
J_{n}(-\ell, a) & =-\frac{1}{2 \pi i} \int_{C_{2}} \frac{(-z)^{-\ell-1} e^{(n-a) z}}{\left(e^{z}+1\right)^{n}} d z \\
& =\frac{(-1)^{\ell}}{2^{n}} \operatorname{Res}_{z=0}\left\{z^{-\ell-1}\left(\frac{2}{e^{z}+1}\right)^{n} e^{(n-a) z}\right\} \\
& =\frac{(-1)^{\ell}}{2^{n} \ell !} E_{\ell}^{(n)}(n-a) .
\end{aligned}
$$

We finally get a relationship between $\eta_{n}(s, a)$ and $E_{n}^{(\alpha)}(a)$.

Corollary 3. The following relationship holds true:

$$
\eta_{n}(-\ell, a)=\frac{(-1)^{\ell}}{2^{n}} E_{\ell}^{(n)}(n-a) \quad\left(\ell \in \mathbb{N}_{0} ; n \in \mathbb{N}\right) .
$$

In particular, for the Hurwitz-Euler eta function $\eta(s, a)$,

$$
\eta(-\ell, a)=\frac{(-1)^{\ell}}{2} E_{\ell}(1-a) \quad\left(\ell \in \mathbb{N}_{0}\right)
$$

in terms of the classical Euler polynomials $E_{k}(a)$.

We conclude this paper by remarking that, as in Section 2, it is not difficult to get a formula for $\eta_{n}(s, a)$ analogous to the assertion (2.1) of Theorem 1. However, we do not find it be worthwhile to try to get such an analogous formula for $\eta_{n}(s, a)$, since $\eta_{n}(s, a)$ is already continued analytically as an entire function of $s \in \mathbb{C}$.

\section{ACKNOWLEDGMENTS}

This research was supported by the Basic Science Research Program through the National Research Foundation of Korea funded by the Ministry of Education, Science and Technology of the Republic of Korea (2010-0011005).

The present investigation was supported, in part, by the Natural Sciences and Engineering Research Council of Canada under Grant OGP0007353. 


\section{REFERENCES}

1. E. W. Barnes, The theory of the G-function, Quart. J. Math., 31 (1899), 264-314.

2. E. W. Barnes, Genesis of the double Gamma function, Proc. London Math. Soc., (Ser. 1), 31 (1900), 358-381.

3. E. W. Barnes, The theory of the double Gamma function, Philos. Trans. Roy. Soc. London Ser. A, 196 (1901), 265-388.

4. E. W. Barnes, On the theory of the multiple Gamma functions, Trans. Cambridge Philos. Soc., 19 (1904), 374-439.

5. J. Choi, Determinant of Laplacian on $S^{3}$, Math. Japon., 40 (1994), 155-166.

6. J. Choi, Explicit formulas for the Bernolli polynomials of order $n$, Indian J. Pure Appl. Math., 27 (1996), 667-674.

7. M. Garg, K. Jain and H. M. Srivastava, Some relationships between the generalized Apostol-Bernoulli polynomials and Hurwitz-Lerch Zeta functions, Integral Transform. Spec. Funct., 17 (2006), 803-815.

8. H. Kumagai, The determinant of the Laplacian on the $n$-sphere, Acta Arith., 91 (1999), 199-208.

9. S.-D. Lin, H. M. Srivastava and P.-Y. Wang, Some expansion formulas for a class of generalized Hurwitz-Lerch Zeta functions, Integral Transform. Spec. Funct., 17 (2006), 817-827.

10. B. Osgood, R. Phillips and P. Sarnak, Extremals of determinants of Laplacians, $J$. Funct. Anal., 80 (1988), 148-211.

11. J. R. Quine and J. Choi, Zeta regularized products and functional determinants on spheres, Rocky Mountain J. Math., 26 (1996), 719-729.

12. H. M. Srivastava and J. Choi, Series Associated with the Zeta and Related Functions, Kluwer Academic Publishers, Dordrecht, Boston and London, 2001.

13. I. Vardi, Determinants of Laplacians and multiple Gamma functions, SIAM J. Math. Anal., 19 (1988), 493-507.

14. A. Voros, Special functions, spectral functions and the Selberg Zeta function, Comm. Math. Phys., 110 (1987), 439-465.

15. E. T. Whittaker and G. N. Watson, A Course of Modern Analysis: An Introduction to the General Theory of Infinite Processes and of Analytic Functions; With an Account of the Principal Transcendental Functions, Fourth edition, Cambridge University Press, Cambridge, London and New York, 1963. 
Junesang Choi

Department of Mathematics

Dongguk University

Gyeongju 780-714

Republic of Korea

E-mail: junesang@mail.dongguk.ac.kr

H. M. Srivastava

Department of Mathematics and Statistics

University of Victoria

Victoria, British Columbia V8W 3R4

Canada

E-mail: harimsri@math.uvic.ca 